(C) 2020, The Authors. Published by FASS Inc. and Elsevier Inc. on behalf of the American Dairy Science Association ${ }^{\circledR}$. This is an open access article under the CC BY-NC-ND license (http://creativecommons.org/licenses/by-nc-nd/4.0/).

\title{
Novel haplotypes responsible for prenatal death in Nordic Red and Danish Jersey cattle
}

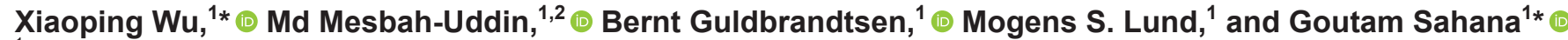 \\ ${ }^{1}$ Center for Quantitative Genetics and Genomics, Faculty of Technical Sciences, Aarhus University, 8830 Tjele, Denmark \\ ${ }^{2}$ Animal Genetics and Integrative Biology, UMR 1313 GABI, INRA, AgroParisTech, Université Paris-Saclay, 78350 Jouy-en-Josas, France
}

\begin{abstract}
Haplotypes that are common in a population but not observed as homotypes in living animals may harbor lethal alleles that compromise embryo survival. In this study, we searched for homozygous-deficient haplotypes in the genomes of 19,309 Nordic Red Dairy (RDC) and 4,291 Danish Jersey (JER) cattle genotyped using the Illumina BovineSNP50 BeadChip (Illumina Inc., San Diego, CA). For statistically significant deficient haplotypes, we evaluated the effect on nonreturn rate in at-risk matings (mating between carrier bull and daughter of carrier sire) versus not-at-risk matings (mating between noncarrier bull and daughter of noncarrier sire). Next, we analyzed whole-genome sequence variants from the 1000 Bull Genomes Project to identify putative causal variants underlying these haplotypes. In RDC, we identified 3 homozygous-deficient regions (HDR) that overlapped with known recessive lethal mutations: a $662-\mathrm{kb}$ deletion on chromosome 12 in RDC [Online Mendelian Inheritance in Animals (OMIA) 001901-9913), a missense mutation in TUBD1, g.11063520T $>$ C, in Braunvieh cattle (OMIA 0019399913), and a 525-kb deletion on chromosome 23 in RDC (OMIA 001991-9913)]. In addition, we identified 15 novel HDR and their tag haplotypes for the underlying causative variants. The tag haplotype located between 39.2 and $40.3 \mathrm{Mbp}$ on chromosome 18 had a negative effect on nonreturn rate in at-risk mating, confirming embryonic lethality. In Danish Jersey, we identified 12 novel HDR and their tag haplotypes for underlying causative variants. For 3 of these 12 tag haplotypes, insemination records of at-risk mating showed a negative effect on nonreturn rate, confirming the association with early embryonic mortality. Cattle that had both genotype and whole-genome sequence data were analyzed to detect the causative variants underlying
\end{abstract}

\footnotetext{
Received November 1, 2019.

Accepted January 27, 2020.

*Corresponding authors: xpwu594419341@gmail.com and goutam. sahana@mbg.au.dk
}

each tag haplotype. However, none of the functional variants or deletions showed concordance with carrier status of the novel tag haplotypes. Carrier status of these detected haplotypes can be used to select bulls to reduce the frequencies of lethal alleles in the population and to avoid at-risk matings.

Key words: embryonic mortality, homozygousdeficient region, recessive lethal, tag haplotype

\section{INTRODUCTION}

The widespread use of individual bulls for AI promotes the propagation of deleterious alleles in the population. Inadvertent mating between carriers of deleterious, recessive alleles may result in the manifestation of fatal phenotypes in the progeny. Mapping of QTL is widely used to define the position of genes that influence disease traits. However, for early embryo loss, it is limited by the challenge of phenotype recording. The availability of large-scale genotype data has enabled the identification of haplotypes with a deficiency of homotypes (VanRaden et al., 2011). Such haplotypes frequently harbor recessive lethal alleles. Thus, breeders can use the haplotype test to avoid matings between carriers for such genetic defects and reduce future frequencies of the lethal alleles. This approach has been shown to be very successful in dairy cattle. For example, several recessive haplotypes have been reported in dairy cattle breeds (VanRaden et al., 2011; Fritz et al., 2013). Some of the causal factors underlying these haplotypes have been identified, such as a nonsense mutation p.Gln579X in the APAF1 gene for haplotype HH1 (Adams et al., 2012), a nonsynonymous mutation p.Phe1135Ser in the SMC2 gene for haplotype HH3 (McClure et al., 2014), a missense mutation p.Asn290Thr in the GART gene for haplotype HH4 (Fritz et al., 2013), and a deletion of $138 \mathrm{kbp}$ for haplotype HH5 in the gene TFB1M (Schütz et al., 2016). In Jersey, a recessive haplotype JH1 has been reported and the underlying factor is a nonsense mutation, p.Arg55*, in the $C W C 15$ gene (Sonstegard et al., 2013). In Brown Swiss, a recessive haplotype BH2 was reported and the underlying factor is a missense 
mutation, p.His210Arg, in the TUBD1 gene (Schwarzenbacher et al., 2016). In French Normande cattle, a recessive haplotype $\mathrm{NH} 2$ and its underlying factor, a missense mutation, p.Tyr452Cys, in the CAD gene, have been reported (Mesbah-Uddin et al., 2019).

In the present study, we exploited genotyping data from a genomic selection program in Nordic Red Dairy Cattle (RDC) and Danish Jersey (JER) population to detect homozygous-deficient regions (HDR) and tag haplotypes (TH) for an underlying causative variant, and examine their effects on nonreturn rate (NRR) as an indicator of early embryo loss. We also scanned available whole-genome sequence (WGS) data to explore putative causative variants.

\section{MATERIALS AND METHODS}

\section{Genotyping and Phasing}

A total of 19,309 RDC were genotyped with Illumina BovineSNP50 BeadChip (Illumina Inc., San Diego, CA). After removing SNP with minor allele frequency $<1 \%$ or a large deviation from Hardy-Weinberg proportions (HWP, $P<10^{-6}$ ), 43,733 SNPs remained for detection of lethal haplotypes. These SNP genotypes were imputed and phased using Beagle 4.0 (Browning and Browning, 2007).

In JER, 4,291 animals were genotyped. After the quality control steps mentioned above for RDC, 39,563 SNP remained for construction of haplotypes by phasing using Beagle 4.0. The SNP were analyzed according to the UMD3.1 assembly of the Bos taurus genome (Zimin et al., 2009), and corresponding positions in the ARS-UCD1.2 cow genome assembly (Schnabel, 2019) were reported in tables. The lift-over to the ARSUCD1.2 genome was based on the end positions of the detected TH.

\section{Detection of HDR and TH for an Underlying Causative Variant}

A 2-step approach was carried out to identify HDR and $\mathrm{TH}$ for an underlying causative variant using phased and imputed genotyped as described earlier (Wu et al., 2019). In the first step, the HDR was defined through the following steps. Haplotypes with a sliding window using 25 consecutive markers for each autosome were constructed. We selected the haplotypes based on 2 conditions: the observed number of homozygotes was $<3$ and there was significant difference between observed and expected number of homozygotes based on chi-squared test and Bonferroni multiple testing correction (threshold for RDC was $P<0.05 / \mathrm{M}_{R D C}=1.06$ $\times 10^{-6}$, where $\mathrm{M}_{R D C}=43,066$; that for JER was $P<$ $0.05 / \mathrm{M}_{J E R}=1.38 \times 10^{-6}$, where $\mathrm{M}_{J E R}=36,358 ; \mathrm{M}$ is the number of haplotypes with a frequency $>0.01$ ). We grouped neighboring homozygous-deficient haplotypes to define a HDR.

In the second step, with variable haplotype length (1-25 markers), the sliding window was moved along each HDR to refine the TH. From each HDR, when available, we selected the haplotype with complete absence of homozygotes as TH; otherwise, we chose the haplotype with lowest $P$-value for the difference between numbers of observed and expected haplotype as TH.

\section{Effect of TH on Insemination Results}

For each TH, the effect on insemination success was evaluated. This was done by estimating the effect on NRR at 35, 56, 100, and $150 \mathrm{~d}$, which was recorded as " 1 " in case of insemination success (i.e., nonreturn, conception) and "0" if the cow returned to service. The data set included 2,970,092 insemination records for RDC and 728,938 for JER. We compared the mating outcomes according to the carrier status of the bull and the sire of the cow. There were 4 classes of matings for each TH: not-at-risk matings (i) noncarrier bull mating with cow of noncarrier sire, and (ii) noncarrier bull mating with cow of carrier sire; and at-risk matings (iii) carrier bull mating with cow of noncarrier sire, and (iv) carrier bull mating with cow of carrier sire. We expected at-risk matings to have lower insemination success than not-at-risk matings. The logistic regression model with mating type of each $\mathrm{TH}$ as fixed effect was analyzed for NRR at 35, 56, 100, or 150 d, separately:

$$
\eta=\mathbf{X}_{\mathrm{p}} \mathbf{p}+\mathbf{X}_{\mathrm{t}} \mathbf{t}+\mathbf{X}_{\mathrm{m}} \mathbf{m}+\mathbf{Z}_{\mathrm{h}},
$$

where $\eta_{i}=\ln \left(\frac{\theta_{i}}{1-\theta_{i}}\right), \theta_{i}$ was the probability of nonreturn for the ith observation, $\mathbf{y}$ was a vector of NRR (" 1 " in case of success and "0" in case of failure) with $\mathbf{y}$ $\sim \operatorname{bin}(n, \boldsymbol{\theta})$, where $n=1, y_{i} \sim B e\left(\theta_{i}\right)$, bin is binomial distribution, and $B e$ is Bernoulli distribution; $\mathbf{p}$ is a vector of effects of parity; $\mathbf{X}_{\mathrm{p}}$ was an incidence matrix relating phenotypes to parity effects; $\mathbf{X}_{t}$ was an incidence matrix relating phenotypes to month-year effect; t was a vector of effects of period-month of insemination (the whole duration was separated into 4 periods: year $\leq 2000$, year 2001-2005, year 2006-2010, and year $>2010$ ); $\mathbf{m}$ was a vector of mating type effects; $\mathbf{X}_{\mathrm{m}}$ was an incidence matrix relating phenotypes to mating type effect for each $\mathrm{TH}$; $\mathbf{h}$ was a vector of random effects of 
herds; and $\mathbf{Z}$ was an incidence matrix relating phenotypes to random herd effects. Analyses were performed using the DMU package (Madsen et al., 2014).

Effects of mating type were investigated by comparing not-at-risk matings $\left(m_{i}\right.$, the elements of $\left.\mathbf{m}\right)$ and atrisk matings $\left(m_{i v}\right.$, the elements of $\left.\mathbf{m}\right)$ using $t$-test with null hypothesis $m_{i}=m_{i v}$, which means the haplotype carrier status has no effect on NRR.

\section{Identification of Candidate Causal Variants}

To identify a point mutation or an insertion/deletion (indel) underlying the detected $\mathrm{TH}$, we examined WGS data for 2,333 cattle (including $74 \mathrm{RDC}$ and 57 JER) from Run6 of the 1000 Bull Genomes Project (Daetwyler et al., 2014) through the following systematic methods. First, we chose variants located within or close to $( \pm 1 \mathrm{Mb})$ each TH. Second, we chose functional variants (deleterious non-synonymous changes, stop gained, stop lost, start lost, and out-of-frame indels) that might be expected to cause a recessive lethal gene defect. Third, we chose variants with an observed number of homozygotes $<3$. Finally, we chose variants that had the same carrier status as the corresponding $\mathrm{TH}$. Here, we expected that if the haplotype tags the causal variant perfectly, then the animals carrying the $\mathrm{TH}$ will be heterozygous for the causal variant, whereas animals not carrying the TH will be homozygous for the reference allele.

Next, we analyzed large deletion calls on 175 WGS animals [67 Nordic Holstein (HOL), 27 JER, and 81 RDC] from Mesbah-Uddin et al. (2018). These deletions were identified using GenomeSTRiP-2 software (Handsaker et al., 2011). A total of 55 RDC and 27 JER animals were in common between the WGS and SNP genotype data set. For these animals, we searched for candidate deletions within $\pm 1 \mathrm{Mb}$ of an HDR. Additionally, to identify large deletions, we analyzed blocks of SNPs deviating from HWP within $\pm 1 \mathrm{Mb}$ of an HDR. Finally, we checked for concordance between the carrier status of the deletion and $\mathrm{TH}$.

\section{RESULTS AND DISCUSSION}

\section{Novel Lethal Haplotypes Detected in RDC}

In RDC, we identified 18 HDR containing $18 \mathrm{TH}$ with a haplotype frequency $>1 \%$ and with, at most, 3 observed homozygotes (Table 1). The frequencies of these haplotypes were between 3.82 and $12.91 \%$. In most situations, we observed more than one homozygote per haplotype, suggesting either incomplete linkage disequilibrium between the haplotype and the causative variant, incomplete penetrance of causative variant in the homozygous state (Fritz et al., 2013), or errors in genotyping or phase reconstruction. Of the 18 detected TH, 3 located on BTA12, BTA19, and BTA23 overlapped with previously reported lethal mutations and the remaining 15 were novel. Among these novel TH, 2 displayed complete absence of homozygotes (Table 1); $1 \mathrm{TH}$ located at BTA18: 39,204,491-40,288,309 bp had a harmful effect on NRR at 4 time points (Figure 2). For the remaining $12 \mathrm{TH}$ (Table 1), at-risk matings did not have a significantly reduced NRR and at least some homozygotes were observed. Therefore, these TH probably were not responsible for early embryo loss. However, they may harbor recessive lethal variants causing embryonic death at later stage (e.g., after 150 d of conception).

Variants in the 1000 Bull Genomes Project Run6 data were examined for concordance with the predicted carrier status of bulls. However, none of the point mutations or indels in the WGS data were concordant with the carrier status of any of these TH. One possible reason is that the causal variant is not in complete linkage disequilibrium with the TH (Wu et al., 2019). Other possible reasons could be that some causal variants are not single nucleotide variants or indels or may have been filtered out the 1000 Bull Genome Project data during quality control (e.g., because of low allele count or low phred score). Another reason could be that we had fewer carriers with WGS because of the limited number of RDC animals and no Danish Jerseys in the 1000 Bull Genomes Project. We also checked deviations from Hardy-Weinberg equilibrium for all SNPs in the pre-edited $50 \mathrm{~K}$ genotype data within each HDR. The minimum $P$-value for SNP within 15 novel HDR was $1.37 \times 10^{-40}$ on BTA14 by using the chi-squared test. However, no large deletion was detected on BTA14. Comparing animals carrying detected deletions using GenomeSTRiP with TH carriers, we validated 2 known deletions: OMIA (Online Mendelian Inheritance in Animals; Lenffer et al., 2006) 001901-9913 and OMIA 001991-9913. No additional large deletions were concordant with carrier status for any other identified TH.

\section{Three Haplotypes Detected in RDC Overlap Known Recessive Lethal Mutations}

OMIA 001901-9913. A 662-kb deletion on chromosome 12 causes embryo death in RDC (Kadri et al., 2014). We detected an HDR at 18.03-24.59 Mb on BTA12 as a recessive lethal region. The corresponding TH with a frequency of $12.91 \%$ located at $18,584,913$ $21,157,380 \mathrm{bp}$ was expected to have 322 homozygotes but only 1 was observed. At-risk matings of this haplotype had significantly lower insemination success compared with not-at-risk matings (Figure 1A). Genotypes 


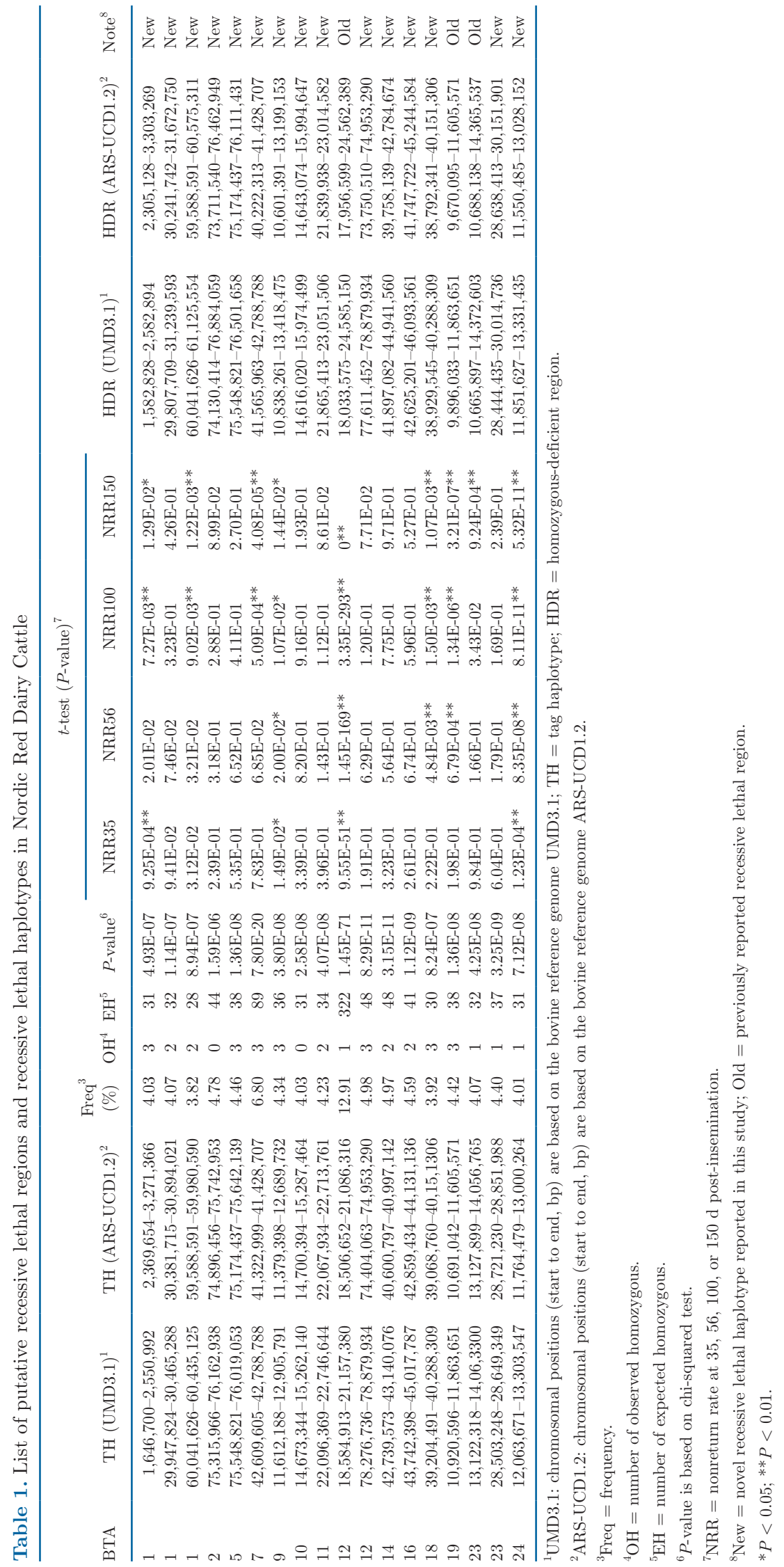


of carrier animals for the markers located within a deletion will be called homozygous for the allele on the haplotype not containing the deletion. This will create an apparent excess of homozygotes, resulting in deviations from HWP. The genotype distribution of SNP in the HDR deviated significantly from HWP, with a minimum $P$-value of $1.90 \times 10^{-42}$. A large chromosomal deletion study (Mesbah-Uddin et al., 2018) contained sequences of $55 \mathrm{RDC}$ animals also present among the genotyped data. Among them, 8 animals were detected to be carriers of the deletion at 20,100,643-20,763,116 bp by using GenomeSTRiP analysis, 7 of which carried the TH. The remaining 47 animals were not carriers of the TH. Therefore, our results show high concordance between $\mathrm{TH}$ and the presence of the deletion.

OMIA 001939-9913 (BH2). On BTA19, we detected an HDR at $9.8-11.9 \mathrm{Mb}$ in RDC. We detected a TH at $10,920,596-11,863,651$ bp on BTA19 with 3 homozygotes where 38 were expected. The frequency of this haplotype was $4.42 \%$. Association analysis validated that at-risk matings of this haplotype had significantly lower NRR at 56,100, and 150 d compared with not-at-risk matings (Figure 1B). Schwarzenbacher et al. (2016) reported a missense mutation (rs383232842, p.H210R) in TUBD1 responsible for juvenile mortality in Braunvieh and Fleckvieh cattle. In the 1000 Bull Genome Project data, 74 RDC overlapped with our genotyped animals and displayed 5 carriers and 69 noncarriers for this TH. If the haplotype tags the causal variant perfectly, we would expect all 5 carriers to be heterozygous for the p.H210R mutation and none of the 69 noncarriers of TH to carry the mutant allele. In contrast, all $5 \mathrm{TH}$ carrier were homozygotes for the reference allele at rs383232842, and 3 carriers of the mutation were noncarriers for the TH. Of note, the p.H210R mutation in TUBD1 is included in the EuroGenomics SNP chip (Boichard et al., 2018) used for routine genotyping in RDC. Only RDC from Denmark had a carrier frequency of $0.2 \%$, and this was not observed among the genotyped RDC animals from Sweden and Finland (details not presented). Therefore, it is highly likely that an additional recessive lethal mutation is segregating in this region in RDC. By comparison with the mouse lethality database of the International Mouse Phenotyping Consortium (IMPC, https://www.mousephenotype.org/), this TH is close to genes SKA2, INTS2, and TBX4 in the IMPC database, which are related to preweaning lethality.

OMIA 001991-9913. On BTA23, we detected an $\mathrm{HDR}$ at $10.6-14.4 \mathrm{Mb}$ in RDC. The TH at $13,122,318$ 14,063,300 bp on BTA23 exhibited 1 homozygote where 32 were expected. The frequency of this haplotype was $4.07 \%$. This region has been previously reported to be related to stillbirth (Sahana et al., 2016; Mesbah-Uddin et al., 2018). However, we observed a significant harmful effect on later NRR (at 100 and $150 \mathrm{~d}$ ), also indicating evidence for prenatal death. Among the 55 RDC animals from Mesbah-Uddin et al. (2018), we detected 5 animals carrying the deletion at 12,291,747-12,817,073 bp on BTA23 by GenomeSTRiP. These 5 deletion carriers were also carriers of the $\mathrm{TH}$, and 50 noncarriers of the deletion were noncarriers of the TH. We observed full concordance between the TH and this deletion.

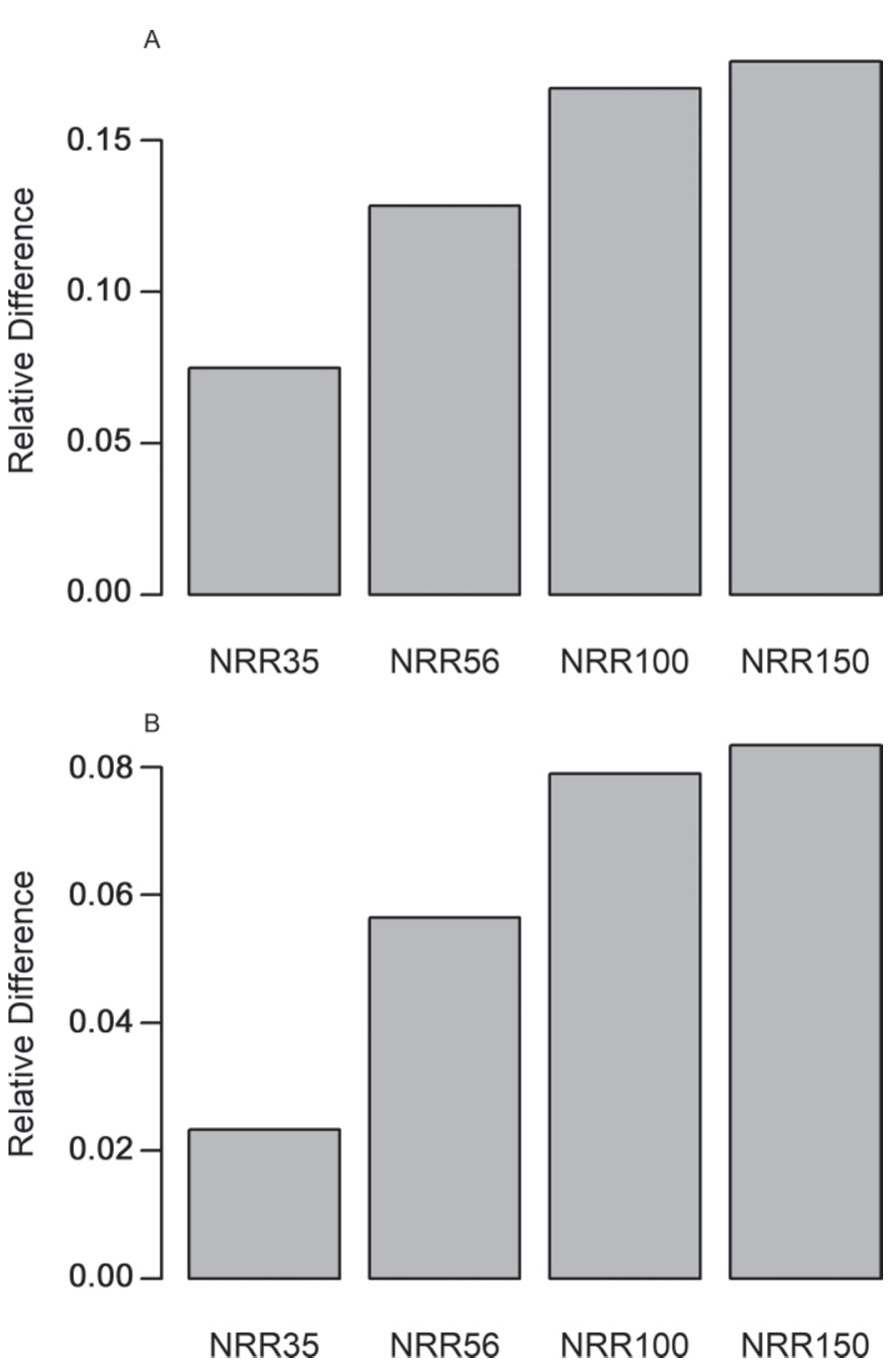

Figure 1. At-risk mating of 2 known lethal haplotypes, Online Mendelian Inheritance in Animals (OMIA) 001901-9913 and OMIA 001939-9913 (BH2), segregating in Nordic Red Dairy Cattle. (A) Haplotype Chr12: 18,033,575-24,585,150 bp; (B) haplotype Chr19: 10,920,596-11,863,651 bp. The y-axis shows the relative difference between at-risk mating (carrier bull mated to a daughter of a carrier sire) and not-at-risk mating (noncarrier bull mated to a daughter of a noncarrier sire), and the $\mathrm{x}$-axis shows the nonreturn rate (NRR) at 35, 56,100 , and $150 \mathrm{~d}$ post-insemination. $\mathrm{Chr}=$ chromosome. 


\section{One Novel Lethal Haplotype Negatively Affecting NRR in RDC}

To confirm the effect of each TH on prenatal death, we compared the effect of not-at-risk matings with at-risk matings, which led to different probabilities of reproductive failure using the logistic regression model. Among 2,970,092 inseminations records analyzed, on average, there were $86.29 \%$ not-at-risk matings [6.27\% of mating type (ii) and $6.74 \%$ of mating type (iii)] and $0.69 \%$ at-risk matings (see Methods for 4 mating types). The average rate of cows that did not return to estrus was 0.28 at $35 \mathrm{~d}, 0.39$ at $56 \mathrm{~d}, 0.47$ at 100 $\mathrm{d}$, and 0.49 at $150 \mathrm{~d}$ after insemination. Of the $15 \mathrm{de}-$ tected novel HDR, at-risk mating of the TH BTA18: 39,204,491-40,288,309 bp showed a significant harmful effect on NRR at $56\left(P=4.48 \times 10^{-3}\right), 100(P=1.50$ $\left.\times 10^{-3}\right)$, and $150 \mathrm{~d}\left(P=1.07 \times 10^{-3}\right)$, supporting the segregation of an embryonic lethal variant tagged by the TH in RDC (Figure 2). The frequency of this haplotype was $3.92 \%$. In the 1000 Bull Genomes Project, there are only 2 carriers for this $\mathrm{TH}$; therefore, we did not perform concordance analysis. According to the IMPC database, this $\mathrm{TH}$ is close to genes $\mathrm{DHODH}$, AP1G1, TAT, and CMTR2, which are related to preweaning lethality.

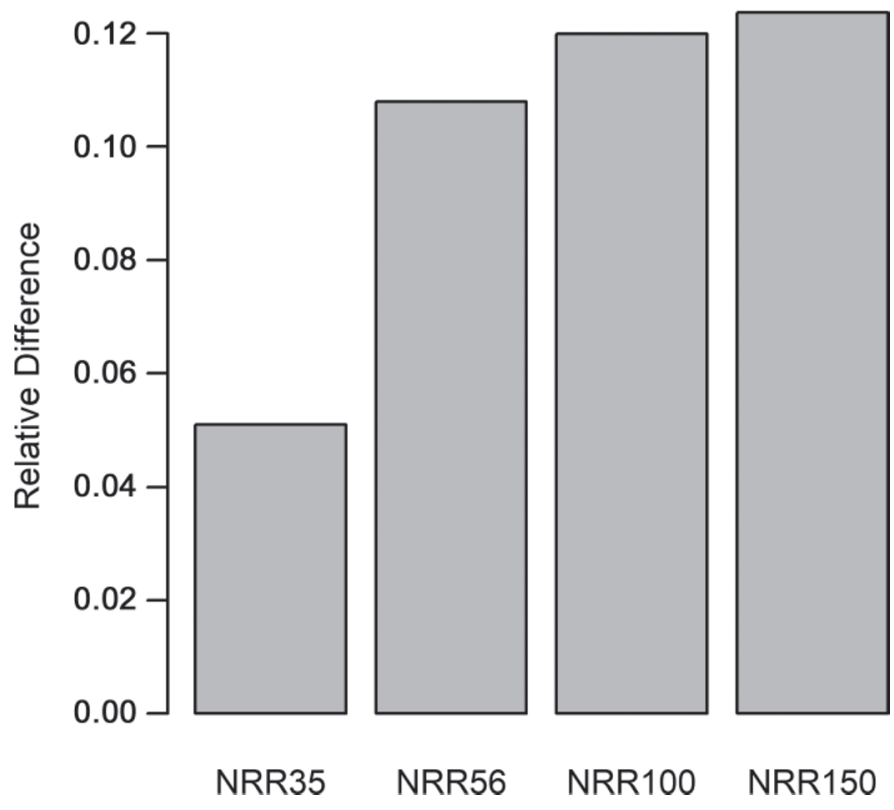

Figure 2. At-risk mating of a novel lethal haplotype Chr18: 39,204,491-40,288,309 bp segregating in Nordic Red Dairy Cattle. The $\mathrm{y}$-axis shows the relative difference between at-risk mating (carrier bull mated to a daughter of a carrier sire) and not-at-risk mating (noncarrier bull mated to a daughter of a noncarrier sire), and the $\mathrm{x}$-axis shows the nonreturn rate (NRR) at 35, 56, 100, and $150 \mathrm{~d}$ post-insemination. $\mathrm{Chr}=$ chromosome

\section{Two Novel Lethal Haplotypes Display Complete Absence of Homozygotes in RDC}

Two TH displaying complete absence of homozygotes signal recessive lethal haplotypes (Table 1). However, these haplotypes did not show harmful effects of atrisk mating compared with not-at-risk matings in NRR to confirm early embryonic losses. A possible reason is that animals carrying these haplotype die later, after $150 \mathrm{~d}$.

\section{Other Novel RDC TH Close to Genes Related to Preweaning Lethality in Mouse}

In the IMPC database, we found 2 genes, SYNJ1 and URB1, located within the TH at BTA1: 1,646,7002,550,992 bp; 1 gene, GAP 43, within the TH at BTA1: 60,041,626-60,435,125 bp; and 4 genes, CASZ1, PEX14, $K I F 1 B$, and $C T N N B I P 1$, within the TH at BTA16: $43,742,398-45,017,787$, all of which were related to preweaning lethality in mouse.

\section{Novel Lethal Haplotypes Detected in Danish Jersey}

We detected 12 HDR in JER population (Table 2). We had power to detect only moderate-frequency haplotypes because the sample size in JER was small compared with that of RDC. We shortlisted $12 \mathrm{TH}$ with 0 to 3 observed homozygous with haplotype frequency $>7.83 \%$. Of these $12 \mathrm{TH}, 3$ had adverse effects on NRR for the at-risk mating. The other $9 \mathrm{TH}$ did not significantly reduce NRR, and one or more homozygotes were observed. The reductions in haplotype homozygosity did not seem to be due to early embryo death. Moreover, by checking the carrier concordance for 57 JER occurring in both genotype data and WGS data, none of the point mutation or indels were concordant with TH carrier status. In addition, no overlapping deletion was detected by GenomeSTRiP. We also checked the HWP for all SNP in the pre-edited 50K genotype data within each HDR. The minimum $P$-value of 5.18 $\times 10^{-17}$ for SNP within the $12 \mathrm{HDR}$ was observed on BTA21. Based on the IMPC database, the TH on BTA7 is close to the gene MGAT1, and the TH on BTA14 is close to the gene $U Q C R B$, both of which are related to preweaning lethality.

\section{Three Novel Lethal Haplotypes Negatively Affecting NRR in JER}

Among 728,938 inseminations records analyzed, on average, there were $70.58 \%$ of not-at-risk matings, $13.34 \%$ of type ii, $13.28 \%$ of type iii, and $2.81 \%$ of atrisk matings. The average rate of cows that did not 


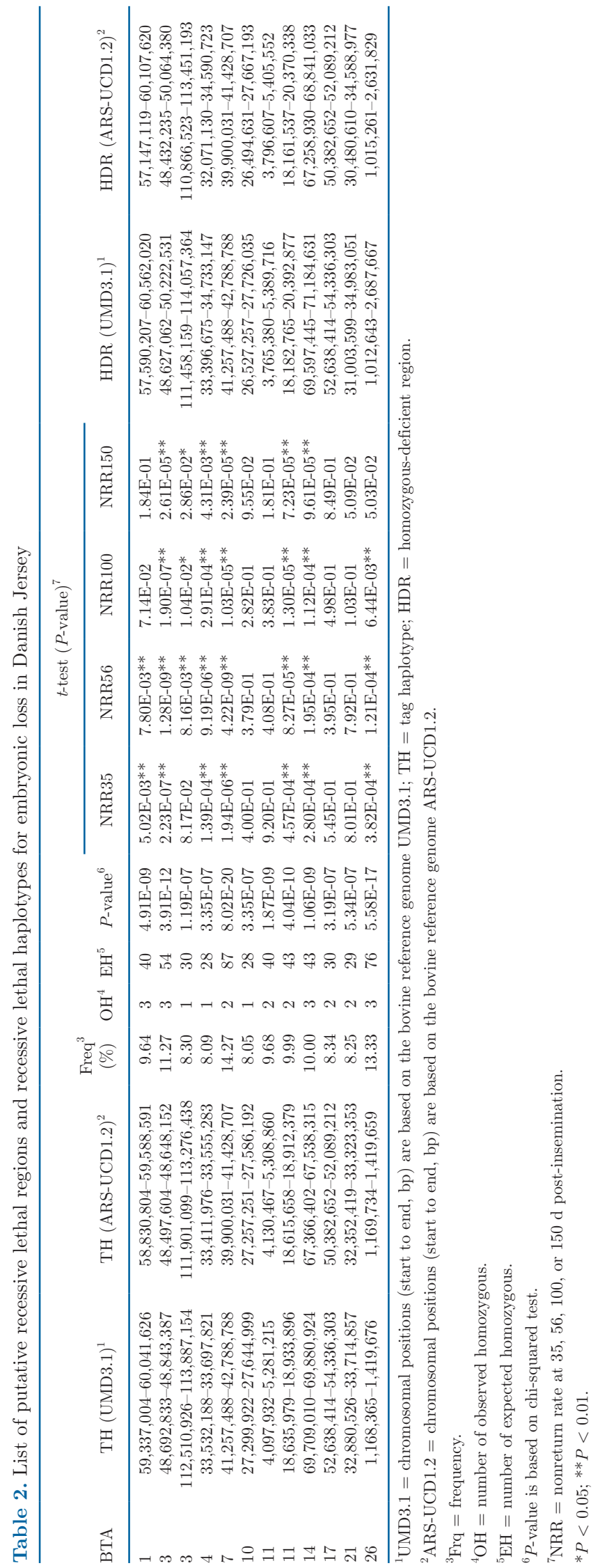

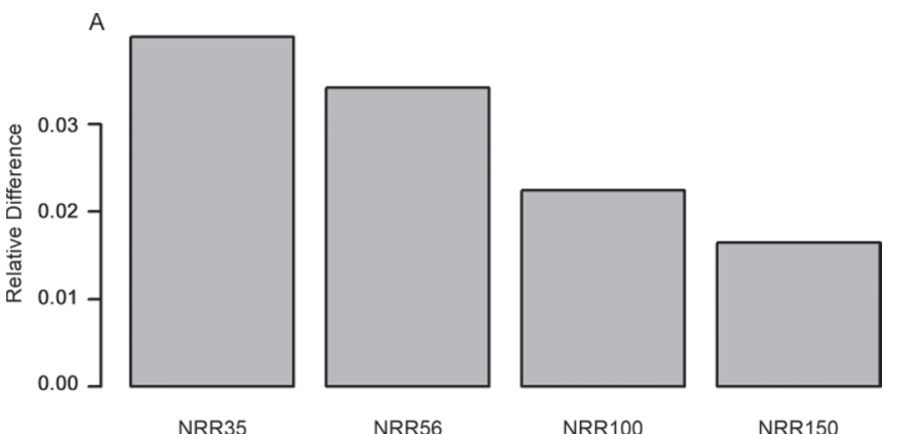
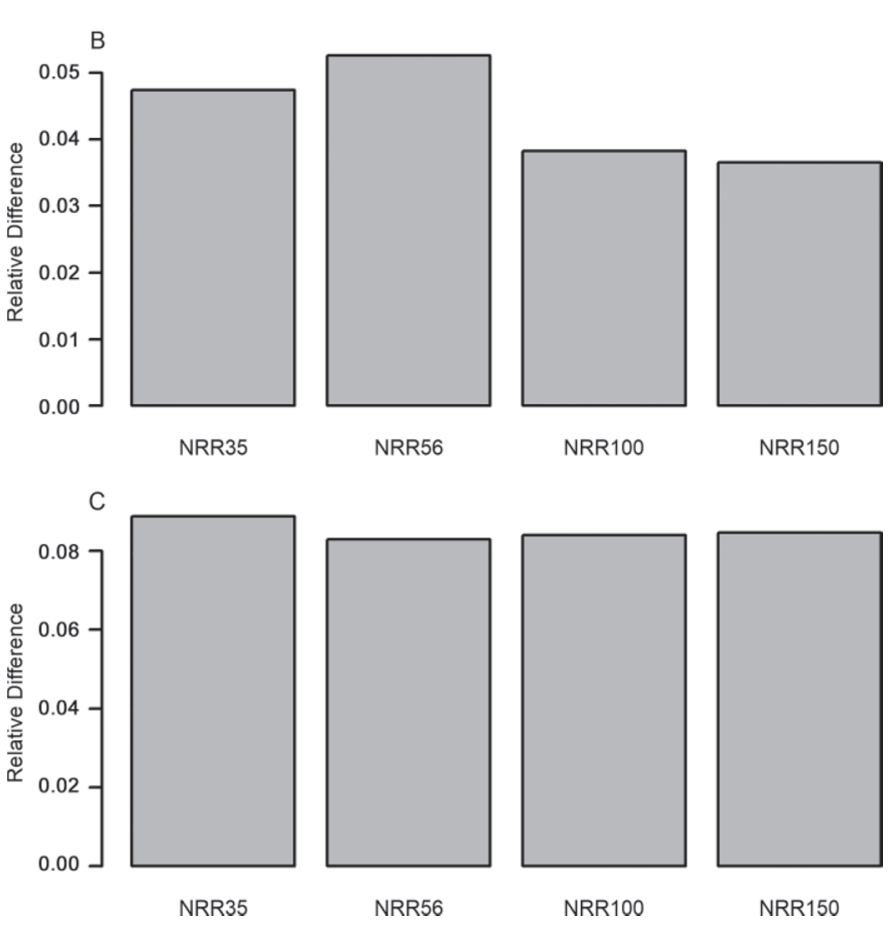

Figure 3. At-risk mating of 3 novel lethal haplotypes segregating in Danish Jersey. (A) Haplotype Chr1: 59,337,004-60,041,626 bp; (B) haplotype Chr7: 41,257,488-42,788,788 bp; and (C) haplotype Chr14: $69,709,010-69,880,924 \mathrm{bp}$. The $\mathrm{y}$-axis shows the relative difference between at-risk mating (carrier bull mated to a daughter of a carrier sire) and not-at-risk mating (noncarrier bull mated to a daughter of a noncarrier sire), and the $\mathrm{x}$-axis shows the nonreturn rate (NRR) at 35 , 56, 100, and $150 \mathrm{~d}$ post-insemination. $\mathrm{Chr}=$ chromosome.

return to estrus was 0.25 at $35 \mathrm{~d}, 0.37$ at $56 \mathrm{~d}, 0.45$ at $100 \mathrm{~d}$, and 0.47 at $150 \mathrm{~d}$ after insemination. Of the 12 detected HDR, at-risk mating of the TH at 59,337,004$60,041,626 \mathrm{bp}$ on BTA1 displayed significant harmful effect on NRR at 35 and $56 \mathrm{~d}$, and at-risk mating of the other $2 \mathrm{TH}$ displayed significant harmful effect on NRR at 35, 56, 100 and $150 \mathrm{~d}$ (Table 2, Figure 3).

\section{Other Novel JER TH Close to Genes Related to Preweaning Lethality in the Mouse}

In the IMPC database, we found 1 gene, ARHGAP29, close to the TH at BTA3: 48,692,833-48,843,387 bp; 2 
genes, KCNJ13 and ATG16L1, within the TH at BTA3: 112,510,926-113,887,154 bp; 1 gene, LIPT1, within the TH at BTA11: 4,097,932-5,281,215 bp; 1 gene, CRIM1, within the TH at BTA11: $18,635,979-18,933,896 \mathrm{bp} ; 4$ genes, UBC, CCDC92, DNAH10, and DDX55, within or close to the TH at BTA17: 52,638,414-54,336,303 bp; and 3 genes, HMG20A, SCAMP5, and CSK, within or close to the TH at BTA21: $32,880,526-33,714,857 \mathrm{bp}$ related to preweaning lethality in the mouse.

\section{Overlap of HDR Among Nordic Holstein, $R D C$, and JER}

Some mutations underlying recessive lethal regions segregating in current populations may have originated before breed separation. These mutations may drift and either became a common allele with high frequency or become rare. In this study, we examined the overlap of the $\mathrm{TH}$ regions detected in RDC, JER, and HOL (Wu et al., 2019). One overlapping HDR was detected at BTA7:41-44 Mb in all 3 breeds; RDC and HOL shared 2 HDR, one at BTA1: 27-29 Mb and another at BTA10: 14-15 Mb. In addition, JER and RDC shared 1 HDR at BTA1: 60-61 Mb, whereas JER and HOL shared one HDR at BTA10: 26-28 Mb. The detected HDR in this study shared by 3 breeds may have originated in the shared gene pool around 300 yr ago, before breed separation (Koolmees and Lenstra, 2014). Most of the HDR were different among the 3 breeds, possibly indicating that most of the HDR currently segregating in the population are of recent origin or were eliminated from some breeds due to drift. Generally, the frequencies of the TH were higher in JER than in RDC and HOL, mainly due to the small sample size of JER, which only allows detection of $\mathrm{TH}$ with moderate frequency.

\section{CONCLUSIONS}

Using genomic data, we detected 18 HDR in RDC of putatively recessive lethal haplotypes based on significant depletion of homozygotes. Three of these haplotypes overlapped with known genetic defects (OMIA 001901-9913, OMIA 001939-9913, and OMIA 001991-9913). Of the 15 novel haplotypes, 1 showed an adverse effect on insemination success and 2 showed complete absence of homozygotes. In JER, we detected 12 novel HDR of putative recessive lethal haplotypes; 3 TH showed an adverse effect on insemination success. Haplotype carrier status could be used in selecting bulls to reduce the frequency of mutations in the population, and at-risk mating could be avoided at the same time. Our findings will enable the implementation of genomebased mating strategies to prevent economic losses.

\section{ACKNOWLEDGMENTS}

We are grateful to the NAV (Aarhus, Denmark) for providing the phenotypic data used in this study and Viking Genetics (Randers, Denmark) for providing blood and semen samples for genotyping. This work was supported by the research projects "Identification and control of recessive mutations" funded by the Milk Levy Fund (Aarhus, Denmark) and the GUDP project LiveCalf (No. 34009-16-1101) from the Ministry of Environment and Food of Denmark. Md Mesbah-Uddin acknowledges the European Commission's ErasmusMundus joint doctorate "EGS-ABG" program and the Center for Genomic Selection in Animals and Plants (GenSAP) funded by Innovation Fund Denmark (grant 0603-00519B). The 1000 Bull Genomes Project is kindly acknowledged for sharing the whole genome sequence data. The authors declare no competing interests.

\section{REFERENCES}

Adams, H. A., T. S. Sonstegard, P. M. VanRaden, D. J. Null, C. P. Van Tassell, and H. A. Lewin. 2012. Identification of a nonsense mutation in APAF1 that is causal for a decrease in reproductive efficiency in dairy cattle. J. Dairy Sci. 99:6693-6701. https://doi .org/10.3168/jds.2015-10517.

Boichard, D., M. Boussaha, A. Capitan, D. Rocha, C. Hoze, M.-P. Sanchez, T. Tribout, R. Letaief, P. Croiseau, C. Grohs, W. Li, C. Harland, C. Charlier, M. S. Lund, G. Sahana, M. Georges, S. Barbier, W. Coppieters, S. Fritz, and B. Guldbrandtsen. 2018. Experience from large scale use of the EuroGenomics custom SNP chip in cattle. Page 675 in Proc. World Congr. Genet. Appl. Livest. Prod. Vol. Molecular Genetics 4, Auckland, New Zealand.

Browning, S. R., and B. L. Browning. 2007. Rapid and accurate haplotype phasing and missing-data inference for whole-genome association studies by use of localized haplotype clustering. Am. J. Hum. Genet. 81:1084-1097. https://doi.org/10.1086/521987.

Daetwyler, H. D., A. Capitan, H. Pausch, P. Stothard, R. van Binsbergen, R. F. Brøndum, X. Liao, A. Djari, S. C. Rodriguez, C. Grohs, D. Esquerré, O. Bouchez, M.-N. Rossignol, C. Klopp, D. Rocha, S. Fritz, A. Eggen, P. J. Bowman, D. Coote, A. J. Chamberlain, C Anderson, C. P. VanTassell, I. Hulsegge, M. E. Goddard, B. Guldbrandtsen, M. S. Lund, R. F. Veerkamp, D. A. Boichard, R. Fries, and B. J. Hayes. 2014. Whole-genome sequencing of 234 bulls facilitates mapping of monogenic and complex traits in cattle. Nat. Genet. 46:858-865. https://doi.org/10.1038/ng.3034.

Felius, M., M.-L. Beerling, D. S. Buchanan, B. Theunissen, P. A. Koolmees, and J. A. Lenstra. 2014. On the history of cattle genetic resources. Diversity 6:705-750. https://doi.org/10.3390/d6040705.

Fritz, S., A. Capitan, A. Djari, S. C. Rodriguez, A. Barbat, A. Baur, C. Grohs, B. Weiss, M. Boussaha, D. Esquerré, C. Klopp, D. Rocha, and D. Boichard. 2013. Detection of haplotypes associated with prenatal death in dairy cattle and identification of deleterious mutations in GART, SHBG and SLC37A2. PLoS One 8:e65550. https://doi.org/10.1371/journal.pone.0065550.

Handsaker, R. E., J. M. Korn, J. Nemesh, and S. A. McCarroll. 2011. Discovery and genotyping of genome structural polymorphism by sequencing on a population scale. Nat. Genet. 43:269-276. https: /doi.org/10.1038/ng.768.

Kadri, N. K., G. Sahana, C. Charlier, T. Iso-Touru, B. Guldbrandtsen, L. Karim, U. S. Nielsen, F. Panitz, G. P. Aamand, N. Schulman, M. Georges, J. Vilkki, M. S. Lund, and T. Druet. 2014. A 660-Kb deletion with antagonistic effects on fertility and milk production segregates at high frequency in Nordic Red cattle: Additional evi- 
dence for the common occurrence of balancing selection in livestock. PLoS Genet. 10:e1004049. https://doi.org/10.1371/journal .pgen.1004049.

Lenffer, J., F. W. Nicholas, K. Castle, A. Rao, S. Gregory, M. Poidinger, M. D. Mailman, and S. Ranganathan. 2006. OMIA (Online Mendelian Inheritance in Animals): An enhanced platform and integration into the Entrez search interface at NCBI. Nucleic Acids Res. 34:D599-D601. https://doi.org/10.1093/nar/gkj152.

Madsen, P., J. Jensen, R. Labouriau, O. F. Christensen, and G. Sahana. 2014. DMU-A package for analyzing multivariate mixed models in quantitative genetics and genomics. Proc. 10th World Congr. Genet. Appl. Livest. Prod., Vancouver, Canada.

McClure, M. C., D. Bickhart, D. Null, P. Vanraden, L. Xu, G. Wiggans, G. Liu, S. Schroeder, J. Glasscock, J. Armstrong, J. B. Cole, C. P. Van Tassell, and T. S. Sonstegard. 2014. Bovine exome sequence analysis and targeted SNP genotyping of recessive fertility defects $\mathrm{BH} 1, \mathrm{HH} 2$, and $\mathrm{HH} 3$ reveal a putative causative mutation in SMC2 for HH3. PLoS One 9:e92769. https://doi.org/10.1371/ journal.pone.0092769.

Mesbah-Uddin, M., C. Hoze, P. Michot, A. Barbat, R. Lefebvre, M. Boussaha, G. Sahana, S. Fritz, D. Boichard, and A. Capitan. 2019. A missense mutation (p. Tyr452Cys) in the CAD gene compromises reproductive success in French Normande cattle. J. Dairy Sci. 102:6340-6356. https://doi.org/10.3168/jds.2018-16100.

Mesbah-Uddin, M., B. Guldbrandtsen, T. Iso-Touru, J. Vilkki, D. J. De Koning, D. Boichard, M. S. Lund, and G. Sahana. 2018. Genome-wide mapping of large deletions and their population-genetic properties in dairy cattle. DNA Res. 25:49-59. https://doi.org/10 $.1093 /$ dnares/dsx037.

Sahana, G., T. Iso-Touru, X. Wu, U. S. Nielsen, D.-J. de Koning, M. S. Lund, J. Vilkki, and B. Guldbrandtsen. 2016. A 0.5-Mbp deletion on bovine chromosome 23 is a strong candidate for stillbirth in Nordic Red cattle. Genet. Sel. Evol. 48:35. https://doi.org/10 .1186/s12711-016-0215-Z

Schnabel, R. 2019. ARS-UCD1.2 Cow genome assembly: Mapping of all existing variants. Accessed Oct. 23, 2019. https://www .animalgenome.org/repository/cattle/UMC_bovine_coordinates/.

Schütz, E., C. Wehrhahn, M. Wanjek, R. Bortfeld, W. E. Wemheuer, J. Beck, and B. Brenig. 2016. The Holstein Friesian lethal hap- lotype 5 (HH5) results from a complete deletion of TBF1M and cholesterol deficiency (CDH) from an ERV-(LTR) insertion into the coding region of APOB. PLoS One 11:e0154602. https://doi .org/10.1371/journal.pone.0154602.

Schwarzenbacher, H., J. Burgstaller, F. R. Seefried, C. Wurmser, M. Hilbe, S. Jung, C. Fuerst, N. Dinhopl, H. Weissenböck, B. FuerstWaltl, M. Dolezal, R. Winkler, O. Grueter, U. Bleul, T. Wittek, R. Fries, and H. Pausch. 2016. A missense mutation in TUBD1 is associated with high juvenile mortality in Braunvieh and Fleckvieh cattle. BMC Genomics 17:400. https://doi.org/10.1186/s12864 -016-2742-y.

Sonstegard, T. S., J. B. Cole, P. M. VanRaden, C. P. Van Tassell, D. J. Null, S. G. Schroeder, D. Bickhart, and M. C. McClure 2013. Identification of a nonsense mutation in CWC15 associated with decreased reproductive efficiency in Jersey cattle. PLoS One 8:e54872. https://doi.org/10.1371/journal.pone.0054872.

VanRaden, P. M., K. M. Olson, D. J. Null, and J. L. Hutchison. 2011. Harmful recessive effects on fertility detected by absence of homozygous haplotypes. J. Dairy Sci. 94:6153-6161. https://doi.org/10 $.3168 /$ jds.2011-4624.

Wu, X., M. Mesbah-Uddin, B. Guldbrandtsen, M. S. Lund, and G. Sahana. 2019. Haplotypes responsible for early embryonic lethality detected in Nordic Holsteins. J. Dairy Sci. 102:11116-11123. https: //doi.org/10.3168/jds.2019-16651.

Zimin, A. V., A. L. Delcher, L. Florea, D. R. Kelley, M. C. Schatz, D. Puiu, F. Hanrahan, G. Pertea, C. P. Van Tassell, T. S. Sonstegard, G. Marçais, M. Roberts, P. Subramanian, J. A. Yorke, and S. L. Salzberg. 2009. A whole-genome assembly of the domestic cow, Bos taurus. Genome Biol. 10:R42. https://doi.org/10.1186/ gb-2009-10-4-r42.

\section{ORCIDS}

Xiaoping Wu $\odot$ https://orcid.org/0000-0002-7844-1397 Md Mesbah-Uddin ( ) https://orcid.org/0000-0003-1846-0411 Bernt Guldbrandtsen (® https://orcid.org/0000-0003-1764-135X Goutam Sahana @ https://orcid.org/0000-0001-7608-7577 